\title{
PROBABILISTIC EVALUATION OF CONTROL SYSTEM ROBUSTNESS
}

\author{
By \\ Robert F. Stengel \\ Laura R. Ray \\ and \\ Christopher I. Marrison
}

IMA Preprint Series \# 1094

January 1993 


\title{
PROBABILISTIC EVALUATION OF CONTROL SYSTEM ROBUSTNESS
}

\author{
ROBERT F. STENGEL, LAURA R. RAY, and CHRISTOPHER I. MARRISON*
}

\begin{abstract}
Practical control systems must operate satisfactorily with uncertain variations in plant parameters (i.e., control systems must be robust), but there are limits to the degree of robustness that may be considered desirable. Tolerance to parameter variations that never occur is not useful, and it could lead to closed-loop systems whose normal performance has been compromised unnecessarily. A probabilistic definition of robustness based on expected parameter variations is consistent with accepted design principles, and it is readily evaluated by simulation. Stochastic Robustness Analysis predicts the effects of likely parameter variations on closed-loop stability and performance through evaluation of commonly accepted criteria. Competing control designs are judged by the likelihood that system response and design metrics will fall within desired bounds. Together with numerical search, probabilistic evaluation is a powerful approach not only for comparing alternative controllers but for designing control systems that satisfy robustness and performance requirements.
\end{abstract}

Key words. control system design and analysis, control system robustness, stochastic methods

AMS (MOS) subject classifications. 93E03, 93E15, 93E23, 93E25

\section{INTRODUCTION}

Practical control systems must be designed to run satisfactorily not only with assumed plant parameters but with possible variations in operating conditions. Perfect models of systems to be controlled (i.e., of plants) are rarely available when controllers are being designed, parameters of similar plants are likely to vary from one example to the next, and dynamic characteristics may change as parts wear or operating points shift. Control system designs must be tolerant of these differences for practical control to take place, that is, they must be robust.

Control system robustness is related to plant uncertainty and is an inherently nonlinear issue, even when the plant and controller are linear. Small variations in plant parameters may have effects that are locally linearizable, allowing robustness to be expressed by conventional stability and performance margins. However, it is easy to postulate systems where robustness criteria based on nominal margins fail (e.g., a two-mode system in which the first mode is certain and determines the stability margin, but the second mode is uncertain and becomes unstable for arbitrarily small parameter variations). In such case, robustness can be expressed only by measures based upon the perturbed system itself, evaluated over the full range of possible variations.

\footnotetext{
* Princeton University, Department of Mechanical and Aerospace Engineering, Princeton, NJ 08544. This research has been supported by the Army Research Office under Contract No. DAAL03-89-K-0092 and the FAA and NASA under Grant No. NGL 31-001-252.

Presented at the IMA Workshop on Control Systems Design for Advanced Engineering Systems: Complexity, Uncertainty, Information and Organization, Minneapolis, MN, Oct. 12-16, 1992.
} 
Measures of control system robustness should be easily understood by control engineers, they should have unambiguous, one-to-one relationships to nominal design goals, and they should be related to what can arguably be known about plant uncertainty. If, for example, a specific degree of stability is required or a step response pattern must be matched, robustness metrics should express the variations in stability and step response that are likely to occur given expectable plant variations.

Robustness to unrealistically large parameter variations may be no more attractive than inadequate robustness, because nominal performance and tolerance of parameter variations tend to produce conflicting design requirements. The degree of robustness required for satisfactory operation is related to the plant variations most likely to occur. This suggests that robustness metrics should be probabilistic, deriving from descriptions of parameter variations that are bounded by manufacturing tolerances or physical constraints. The need for handling the nonlinear effects of uncertainty also suggests that evaluation of robustness should be numerical rather than analytic. Consequently, robustness criteria developed for linear systems are readily extended to nonlinear systems.

Stochastic Robustness Analysis (SRA) uses statistical descriptions of parameter uncertainty and numerical evaluation to determine whether stability and performance robustness criteria are met. This paper presents an overview of the SRA method, as well as examples of its application. These include evaluations of Doyle's linear-quadratic-Gaussian (LQG) counter-example, control systems for aircraft flight, and a recent benchmark problem for robust control system design. One important conclusion of the benchmark-compensator analysis is that there is little correlation between classical stability margins and the likelihood that plant parameter variations lead to instability. The principle reason is that parameter variations change the shape of the Nyquist plot as well as the gain and phase margins; hence, the branch of the nominal Nyquist plot or critical frequency that determines stability margins may not be the one that produces instability when parameters vary. It is further shown that numerical search to minimize stochastic robustness criteria is a practical design method.

\section{ANALYTICAL FOUNDATIONS}

Stochastic stability theory provides a logical starting point, as satisfactory stability is often a necessary condition for satisfactory performance. A typical problem is to determine bounds on the parameter vector $\mathbf{p}$ of an unforced, continuous-time system [1, 2],

$$
\dot{\mathbf{x}}(t)=\mathbf{f}[\mathbf{p}(t), \mathbf{x}(t)], \quad \mathbf{x} \in R^{n}, \mathbf{x}(0)=\mathbf{x}_{0}, \mathbf{f} \in R^{n}, \mathbf{p} \in R^{l}
$$


where $\mathbf{x}$ is the dynamic state and $\mathbf{p}(t)$ is a random process, such that stability can be expected with a probability of one (or arbitrarily close to one). A corresponding linear control problem is to find a satisfactory control gain matrix $\mathbf{C}$ for the linear plant and control law,

$$
\begin{aligned}
& \dot{\mathbf{x}}(t)=\mathbf{F}[\mathbf{p}(t), t] \mathbf{x}(t)+\mathbf{G}[\mathbf{p}(t), t] \mathbf{u}(t), \mathbf{u} \in R^{m}, \mathbf{F} \in R^{n \times n}, \mathbf{G} \in R^{n \times m} \\
& \mathbf{u}(t)=-\mathbf{C x}(t), \mathbf{C} \in R^{m \times n}
\end{aligned}
$$

The system dynamics vector $f(\bullet)$ becomes

$$
\mathbf{f}[\mathbf{p}(t), \mathbf{x}(t)]=\{\mathbf{F}[\mathbf{p}(t), t]-\mathbf{G}[\mathbf{p}(t), t] \mathbf{C}\} \mathbf{x}(t)
$$

and the uncertainty is contained in the varying values of $[\mathbf{F}(\bullet), \mathbf{G}(\bullet)]$. Probabilistic stability criteria have been developed using expectations of Lyapunov functions, and they require consideration of stochastic integrals and transformations [3, 4]. Analogous discrete-time problems are discussed in [5]. Given infinite (e.g., Gaussian) parameter distributions, the probability of instability is finite, and the escape (or exit) time may be of interest [6].

The principal focus of current robustness research is on ensembles of linear systems for which

$$
\begin{aligned}
\mathbf{f}(\mathbf{p}, \mathbf{x}, \mathbf{t}) & =\mathbf{F}^{\prime}(\mathbf{p}) \mathbf{x}(\mathbf{t}) \\
& =[\mathbf{F}(\mathbf{p})-\mathbf{G}(\mathbf{p}) \mathbf{C}] \mathbf{x}(t)
\end{aligned}
$$

where $\mathbf{p}$ is a random constant rather than a random process. For a particular parameter value $\mathbf{p}_{\mathrm{k}}, \mathbf{F}^{\prime}\left(\mathbf{p}_{\mathrm{k}}\right)$ is uncertain but fixed. Deterministic stability criteria apply to each member of the ensemble. Because each dynamic system in eq. 2.5 is linear and time-invariant, its stability is entirely determined by its eigenvalues, that is, the solutions $\lambda_{j}$ to the equation

$$
\left|\lambda_{\mathbf{j}} \mathbf{I}-\mathbf{F}^{\prime}\left(\mathbf{p}_{\mathbf{k}}\right)\right|=0, \mathbf{j}=1 \text { to } n
$$

Given a vector of the probability density functions of $\mathbf{p}, \operatorname{pr}(\mathbf{p})$, eq. 2.6 provides an implicit transformation for computing the probability density functions, $\operatorname{pr}\left(\lambda_{\mathrm{j}}\right)$, of the corresponding ensemble of eigenvalues $\lambda_{j}, j=1$ to $n$. An evaluation of the cumulative probability of (in)stability induced by $\operatorname{pr}(\mathbf{p})$ requires integration of the $\operatorname{pr}\left(\lambda_{j}\right)$ over the (right) left-half complex plane. Linear eigenvalue sensitivities, $\partial \lambda_{j} / \partial \mathbf{p}$, can be derived and applied 
for analytic evaluation of the integral [7, 8], and additional studies of eigenvalue and eigenvector sensitivities can be found in [9-15].

Unfortunately, analytical solutions to this integral have limited utility for evaluating the probability of (in)stability. The relationship between $\mathbf{p}$ and $\lambda_{\mathrm{j}}$ is decidedly nonlinear, so $\partial \lambda_{j} / \partial \mathbf{p}$ does not provide enough information about variations if the domain of $\mathbf{p}$ is large. For system order greater than one (excluding trivial cases), the nonlinear mapping is not the only problem: there is no clear way of integrating the probability density functions to obtain the cumulative probability of (in)stability because $a$ ) roots coalesce and split as parameters change, and $b$ ) instability is the result of one or more roots having positive real components. A further complication is that the combined effects of multiple non-Gaussian parameters cannot be represented merely by operations on mean values and covariances. We conclude that the most practical approach for evaluating the probability of (in)stability in the general case is to use numerical computation, as expanded below.

Numerical evaluation of probabilities involves sampling of parameter probability distributions $[16,17]$ and computation of their consequences using either exhaustive sampling or "Monte Carlo" methods [18]. In the first case, all possible parameter combinations in a finite set are sampled, and the exact probability of hypothesis $\mathcal{H}$ is computed as

$$
\operatorname{Pr}(\mathcal{H})=N_{\mathcal{H}} N_{\text {Total }}
$$

where $N_{\mathcal{H}}$ is the number of instances of $\mathcal{H}$, and $N_{\text {Total }}$ is the total number of trials. For the second method, each scalar parameter is represented by a random number generator, whose characteristics are shaped by the parameter's statistical description. There is no restriction on the shapes or correlations of probability distributions (i.e., they may be bounded, nonGaussian, etc.), and parameters may have different distribution types. For a single trial, each element of $\mathbf{p}_{\mathbf{k}}$ is generated, and the related hypothesis (in the current discussion, the stability or instability of the controlled system) is computed. The probability of a hypothesis is computed as before (eq. 2.7), but there is uncertainty in the estimate, as discussed below. Monte Carlo simulation finds widespread use in risk and reliability assessment (e.g., [19, 20]), but its use in control system assessment has been limited [21, 22]. Application of Monte Carlo methods to evaluating the stability and performance robustness of control systems is recent $[13,15,23-30]$. The approach is seen as a way of confirming robustness estimates made by other techniques (e.g., [31, 32]).

Probabilistic synthesis of control systems is a natural adjunct to probabilistic analysis; the random or randomized search is a dual to Monte Carlo evaluation. Building on [33], random-search methods of finding control system gains are explored in [34-36]. There are 
similarities to directed searches that minimize multi-objective cost functions [37], to parameter-space methods [38, 39], and to fine-tuning of control gains by search [40]. A genetic algorithm -- which performs randomized reproduction, crossover, and mutation on candidate control-gain strings -- has been used to design controllers [41], while SRA is extended to control design using sequential line searches in [23-29, 42].

\section{EVALUATION OF ROBUSTNESS}

Attention is directed to the stability robustness of linear, time-invariant (LTI) systems, for which the probability of instability $\mathbb{P}$ can be estimated from repeated eigenvalue calculation [25]. Given a system with $l$ parameters, each of which takes $w$ values with equal probability, $\mathbb{P}$ can be calculated exactly from $w^{l}$ evaluations using exhaustive sampling (eq. 2.7 ), with $N_{\mathcal{H}}$ equal to the number of unstable cases, and $N_{\text {Total }}$ equal to $w^{l}$. Even if the parameters are binary $(w=2)$, the number of evaluations quickly becomes large as the number of parameters increases. Note that $2^{l}$ also is the number of corners in a bounded parameter space, suggesting that any robustness test that examines the corners has practical limitations to the number of parameters that can be evaluated. For small numbers of parameters, it may be most efficient to approximate continuous distributions by $l$ discrete values, then to apply exhaustive sampling to estimate $\mathbb{P}$ with the discrete distributions.

For Monte Carlo evaluation, the closed-loop eigenvalues, $\lambda_{\mathrm{j}}$, are evaluated $N_{\text {Total }}$ times with each element of $\mathbf{p}_{\mathrm{k}}, \mathrm{k}=1$ to $N_{\text {Total }}$, specified by a random number generator whose individual outputs are shaped by $\mathbf{p r}(\mathbf{p})$. The probability-of-(in)stability estimate becomes increasingly precise as $N_{\text {Total }}$ becomes large:

$$
\begin{aligned}
& \text { (3.1) } \operatorname{Pr}(\text { stable })=\lim _{N_{\text {Total }} \rightarrow \infty} \frac{N\left(\sigma_{\max } \leq 0\right)}{N_{\text {Total }}} \\
& \text { (3.2) } \operatorname{Pr}(\text { unstable }) \quad=\mathbb{P}=1-\operatorname{Pr}(\text { stable })
\end{aligned}
$$

$N(\bullet)$ is the number of cases for which all elements of $\underset{\sim}{\sigma}$, the vector of the real parts of the closed-loop eigenvalues $(\lambda=\sigma+j \omega)$, are less than or equal to zero, that is, for which $\sigma_{\max } \leq 0$, where $\sigma_{\max }$ is the maximum real eigenvalue component in $\underset{\sim}{\sigma}$. For $N_{\text {Total }}<\infty$, the Monte Carlo evaluation is an estimate, $\hat{\mathbb{P}}$, whose uncertainty is characterized by a confidence interval.

Because $\mathbb{P}$ is a binomial variable (i.e., the outcome of each trial takes one of two values: stable or unstable), confidence intervals are calculated using the binomial test, where lower $(\mathcal{L})$ and upper $(\mathcal{U})$ intervals satisfy the following [43]: 
(3.3) $\operatorname{Pr}\left(N_{U} \leq \mathrm{n}-1\right)=\sum_{\mathrm{k}=0}^{\mathrm{n}-1}\left(N_{\text {Total }}, \mathrm{k}\right) \mathcal{L}^{\mathrm{k}}(1-\mathcal{L})^{N_{\text {Total }}}-\mathrm{k}=1-\frac{\alpha}{2}$

(3.4) $\operatorname{Pr}\left(N_{U} \leq \mathrm{n}\right)=\sum_{\mathrm{k}=0}^{\mathrm{n}}\left(N_{\text {Total }}, \mathrm{k}\right) u^{\mathrm{k}}(1-\mathcal{u})^{N_{\text {Total }}}-\mathrm{k}=\frac{\alpha}{2}$

$N_{U}$ is the actual number of unstable cases after $N_{\text {Total }}$ evaluations $\left(N_{U}=N_{\text {Total }} \hat{\mathbb{P}}\right)$, $\left(N_{\text {Total }}, \mathrm{k}\right)$ is the binomial coefficient, $\frac{N_{\text {Total }} !}{\mathrm{k} !\left(N_{\text {Total }}-\mathrm{k}\right) !}$, and $(1-\alpha)$ is the confidence coefficient. Explicit approximations of the binomial test $[44,45]$ avoid an iterative solution of eq. 3.3 and 3.4 for $(\mathcal{L}, \mathcal{U})$, and they are accurate to within $0.1 \%$ [25].

The number of evaluations required to estimate a binomial probability distribution for specified interval widths and a $95 \%$ confidence coefficient varies with the true $\mathbb{P}$ (Fig. 1) [23]. For narrow intervals and small $\mathbb{P}$, large numbers of evaluations are required; however, large percentage interval widths may be acceptable if $\mathbb{P}$ is small.

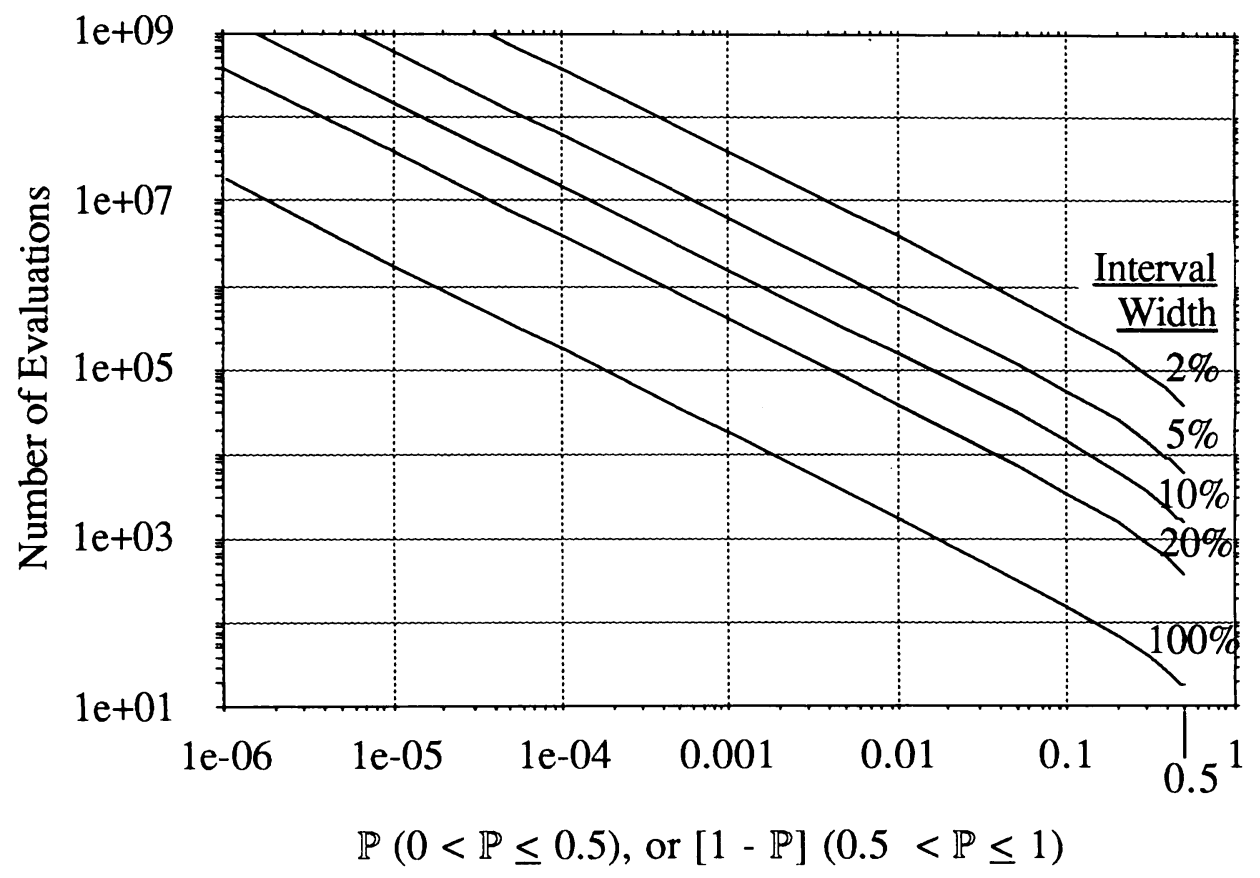

Figure 1. Number of evaluations required to estimate a binomial probability distribution for given confidence interval widths and $95 \%$ confidence coefficient. Interval width is given as percent of $\mathbb{P}$ or $(1-\mathbb{P})$. (from [23])

The number of Monte Carlo evaluations needed to yield $\hat{\mathbb{P}}$ with a given confidence level is independent of the number of uncertain parameters or their probability distributions. 
This result has broad implications for the robustness evaluation of complex systems. While exact or approximate exhaustive sampling may be useful when there are few parameters, Monte Carlo simulation has broad application for systems with large numbers of uncertain parameters.

3.1 Flight Control Example. Evolution of probability estimates typically provides useful information after a few hundred trials, and it is possible to compare competing controllers well before estimates have converged to small confidence intervals (Fig. 2). Three linear-quadratic (LQ) control laws for an open-loop-unstable aircraft are compared, given large uncertainties in a dozen system parameters [24]. Unusually fast response and large uncertainties were specified in these examples to magnify robustness issues. From the beginning, it is clear that Case (a) is less robust than the other controllers, and the distinctions between Cases (b) and (c) are outside the individual error bands after less than 2000 trials. (This is a conservative result, as shown in [26].) After a few hundred evaluations, subsequent probability estimates are seen to fall within the error bands predicted earlier.

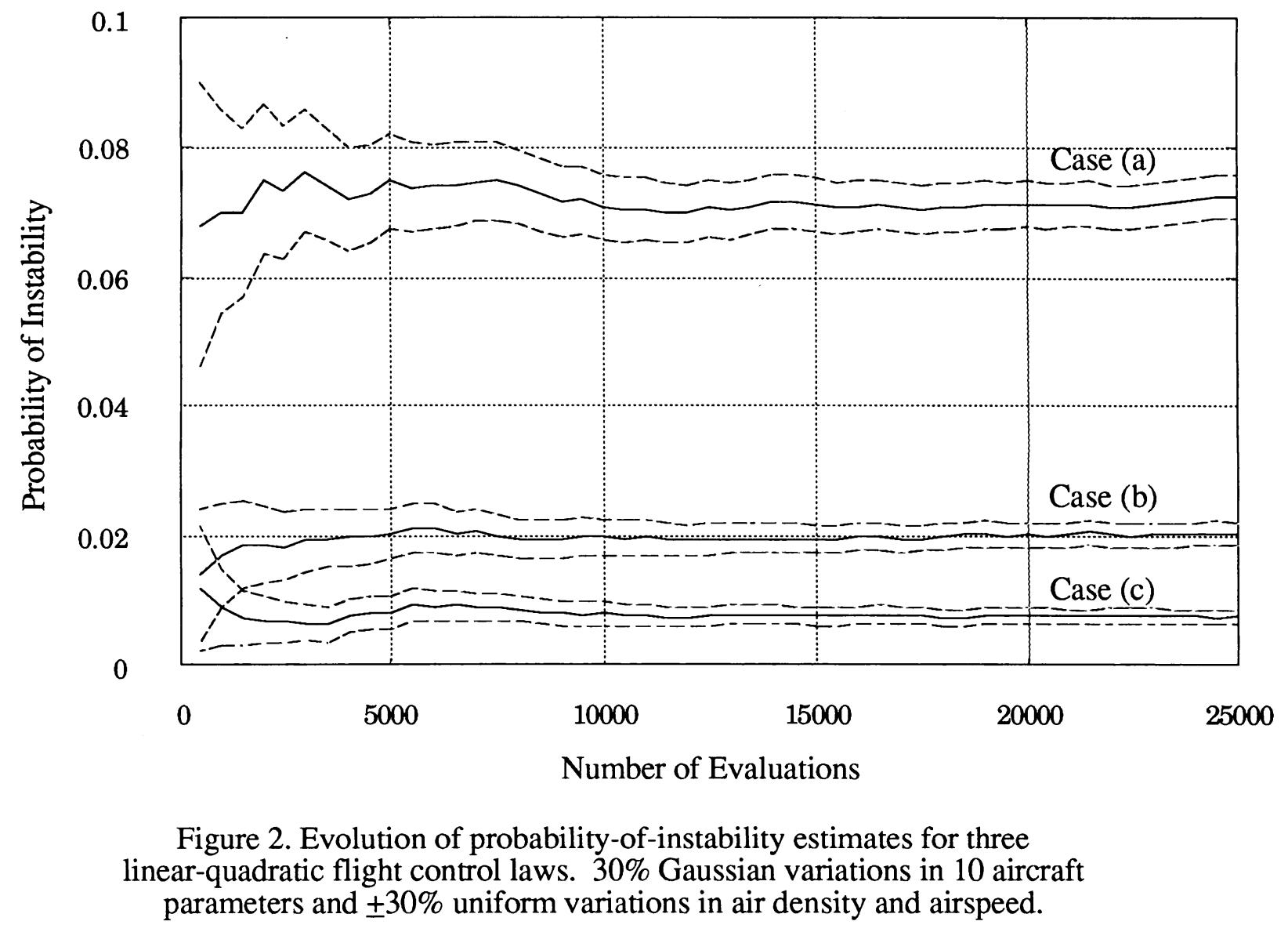


Retaining the two most significant rigid-body parameters (control sensitivity and static stability coefficients) while adding a dozen aeroelastic (fuselage bending and wing torsion) parameters produces the results shown in Fig. 3. The three controllers are unchanged from the previous example, so the aeroelastic modes represent unknown high-frequency dynamics not accounted for in control design. To demonstrate the consistency of probability estimates, the number of evaluations has been increased from 25,000 to 100,000 . What had been the most robust controller [Case (c)] is now almost as likely to produce instability as the least robust controller in the previous example. Case (b) is revealed to be the most robust controller early in the evaluation. Exhaustive sampling of 16 binary parameters would have required 65,536 evaluations; if each parameter were free to take five values, the required number of exhaustive trials increases to over 152 billion. Monte Carlo evaluation produces useful results with considerably fewer samples and allows parameters to have continuous distributions.

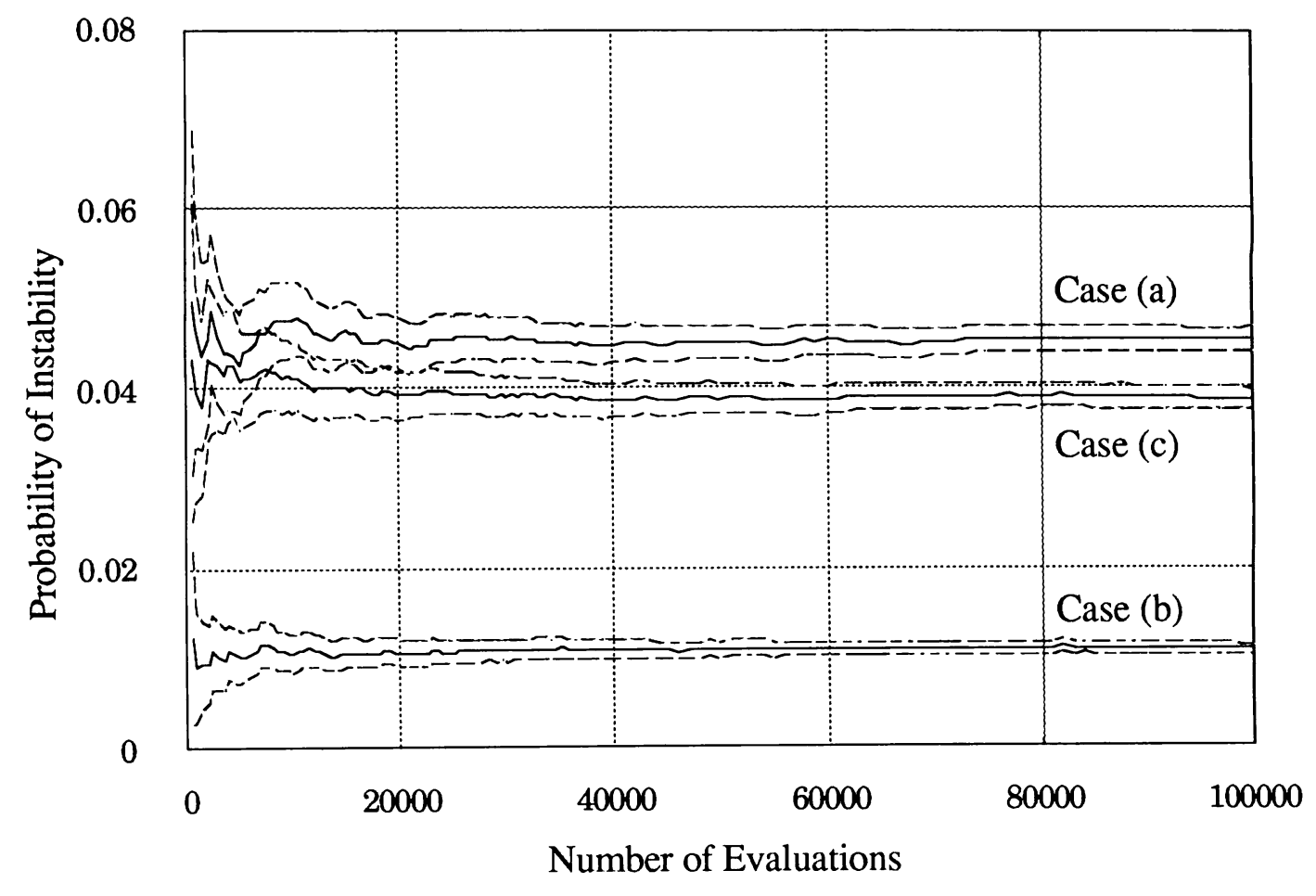

Figure 3. Evolution of probability-of-instability estimates for three linear-quadratic flight control laws, with aeroelastic modes. $30 \%$ Gaussian variations in 2 most significant rigidbody and 12 aeroelastic parameters, $\pm 30 \%$ uniform variations in air density and airspeed.

Various ancillary graphs provide important insights into the nature of robustness, including histograms and cumulative distributions of $\sigma_{\max }$, stochastic root loci, and parameter plots [23]. Histograms and cumulative distributions of maximum eigenvalues 
show the most likely values of the least-stable time constant (which can be related to times to half or double), and they suggest that probabilities of degrees of instability can be investigated by choosing discriminants other than neutral stability $(\sigma=0)$. Alternatively, histograms of parameter values associated with instability identify "trouble spots" requiring additional attention in the design process. Stochastic root loci illustrate concentrations of eigenvalues that result from parameter variations, either as cluster plots of individual eigenvalues or as topographical root density heights above the $s$ plane. These are particularly useful for identifying critical frequencies at which instabilities are most likely to occur. They portray the migration of roots from real to complex modes and sometimes reveal counter-intuitive results, such as peak root densities displaced from the nominal root locations. Because system specifications often are given as sector bounds in the $s$ plane, the degree to which these bounds may be violated is readily shown. Differences between Gaussian and uniform parameters are seen as fuzzy vs. sharp bounds in root locations [2329].

When one or two adjustable control parameters appear (e.g., a scalar weight on an LQ control weighting matrix $\rho \mathbf{R}$ or diagonal elements of $\mathbf{R}$ itself), plotting $\hat{\mathbb{P}}$ against the parameter(s) may reveal a region of maximum robustness that is relatively insensitive to the parameters' distribution types [23]. Such plots also can uncover regions of conditional robustness at intermediate control-parameter values.

3.2 Doyle's LQG Counter-Example. Early misconceptions about the robustness of LQG regulators were shattered by [46], which presented an example that could be driven to instability by arbitrarily small parameter variation as weights on state-regulation error and disturbance-input covariance become large. While this paper set the stage for later development of loop transfer recovery (LTR) [47] and more complete studies of LQG robustness [48], a widespread notion lingers that LQG regulators are, by their nature, not robust. As shown in Section 4, LQG regulators can be made exceedingly robust by choosing cost-function weighting matrices to minimize stochastic robustness metrics.

To briefly review the example [46], an open-loop-unstable, second-order plant is subjected to scalar control through a control-effect multiplier $\mu$ (nominally equal to 1 ) and is observed by measurement of a single state element. The LQG control and estimator gain matrices are

$$
\begin{array}{ll}
\mathbf{C}=\left(2+\sqrt{4+\mathrm{Q})}\left[\begin{array}{ll}
1 & 1
\end{array}\right]\right. & \triangleq\left[\begin{array}{ll}
c & c
\end{array}\right] \\
\mathbf{K}=\left(2+\sqrt{4+\mathrm{W})}\left[\begin{array}{ll}
1 & 1
\end{array}\right]^{\mathrm{T}}\right. & \triangleq\left[\begin{array}{ll}
k & k
\end{array}\right]^{\mathrm{T}}
\end{array}
$$


where $\mathrm{Q}$ and $\mathrm{W}$ scale state-error and disturbance effects for unit control and measurementerror effects. The corresponding characteristic equation

$$
\left[s^{4}+c_{3} s^{3}+c_{2} s^{2}+(k+c-4-2 c k) s+(1+c k)\right]+\mu c k(2 s-1)=0
$$

takes a root-locus form $\left[\approx \mathrm{D}(s)+\mathrm{K}_{\mathrm{L}} \mathrm{N}(s)\right.$ ] with $\mu c k$ serving as the loop gain $\mathrm{K}_{\mathrm{L}}$ [28]. There is a numerator zero at $+1 / 2$, and the product of the control and estimator gains has a direct effect on loop gain. There are five unstable branches for loop gain in $(-\infty, \infty)$. With positive $c_{2}$ and $c_{3}$, the Routh criterion, a necessary (but not sufficient) condition for stability, requires that

$$
a \triangleq\left[1+\frac{1}{c k}\right]<\mu<\left[1-\frac{k+c-4}{2 c k}\right] \triangleq b
$$

The interval $(a, b)$ becomes small as $c k$ increases.

Letting $\mathrm{Q}=\mathrm{W}$ (giving $c=k$ ), $c$ approaches 4 as $\mathrm{Q}$ approaches zero (Case 1 ), and the system's four eigenvalues are located at -1 when $\mu=1$. When $c=k=100$ (Case 2), there are double eigenvalues at -0.01 and -98 with $\mu=1$. For Case $1,(a, b)$ is $(0.875,1.0625)$, and for Case 2 it is $(0.9902,1.0001)$. Eigenvalue calculations indicate that the actual bounds on $\mu$ are $(0.9243,1.0625)$ for Case 1 and $(0.9903,1.0001)$ for Case 2 [28]. If $\mu$ is a Gaussian random variable with mean of one and standard deviation of 0.05 (assuring that some values of the $\mu$ distribution lie outside the stable interval), 10,000-sample Monte Carlo evaluations estimate $\mathbb{P}$ as 0.171 for Case 1 and 0.919 for Case 2 .

The corresponding stochastic root loci (Fig. 4) show the probabilistic root distributions, giving a qualitative picture of the most likely locations of roots and indicating the frequencies at which instability occurs. (The actual traces are lines rather than volumes in this single-parameter case, the jagged "mountains" being artifacts of summing in rectangular "bins" of the $s$ plane and of the graphing process.) Histograms show the most likely regions for $\sigma_{\max }$ in the two cases, while cumulative distributions illustrate not only the probability of stability (at the zero crossing) but the probabilities of intermediate degrees of stability or instability (Fig. 5).

LQG robustness can be recovered to the level of the corresponding LQ regulator using loop transfer recovery [47], which entails modifying the disturbance input spectral density matrix as follows:

$$
\mathbf{W}^{\prime}=\mathbf{W}+v^{2} \mathbf{G G}^{\mathrm{T}}
$$




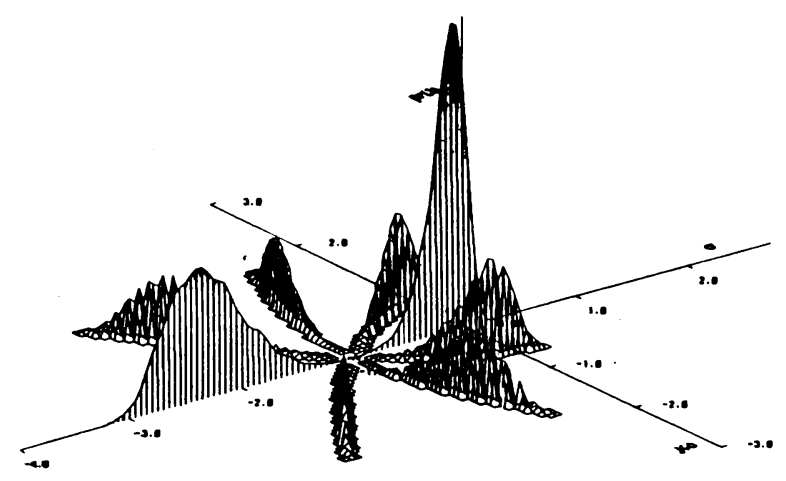

a) Case 1 .

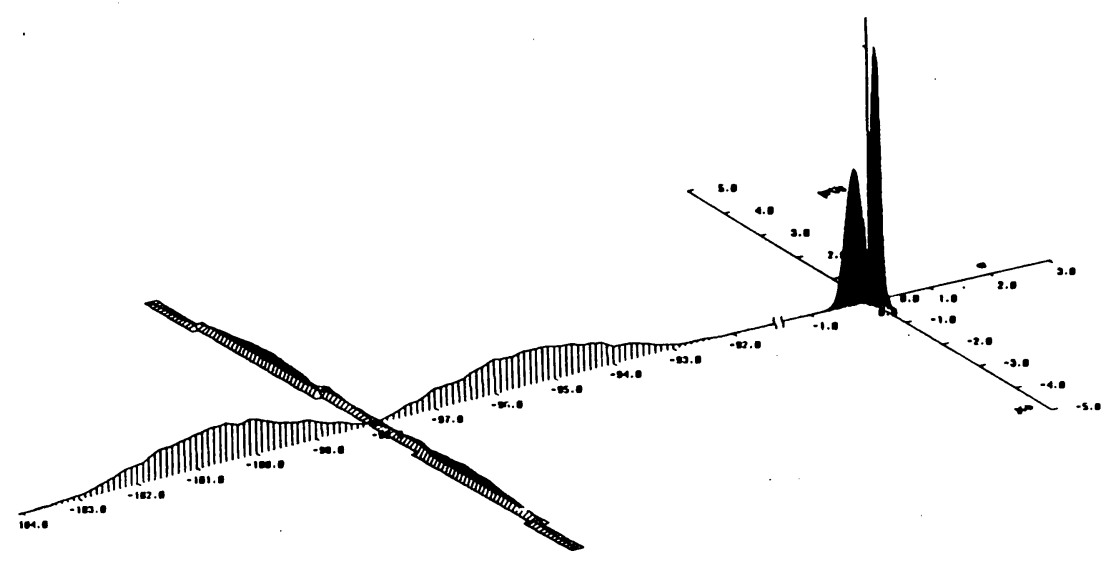

b) Case 2 .

Figure 4. Stochastic root loci for LQG counterexample, 10,000 samples, $\bar{\mu}=1, \sigma_{\mu}=0.05$. (from [28])

The LQ regulator corresponding to Case $1(c=4)$ is completely stable over 10,000 trials with the Gaussian $\mu$ used above. Singular-value analysis suggests that $v$ must be greater than 10,000 for LQ transfer characteristics to be restored to the LQG regulator; however, SRA shows that complete stability over 10,000 trials is recovered for a $v$ of 5 [28]. The stochastic root loci of the LQ regulator and the LQG/LTR regulator with $v=5$ are compared in Fig. 6. The comparison reveals the estimator roots as a complex pair that never threaten instability but that push the controller roots to the right. Increasing values of $v$ force the estimator to higher frequency and let the controller roots move closer to the LQ locations, at the cost of increased transmission of measurement noise. 

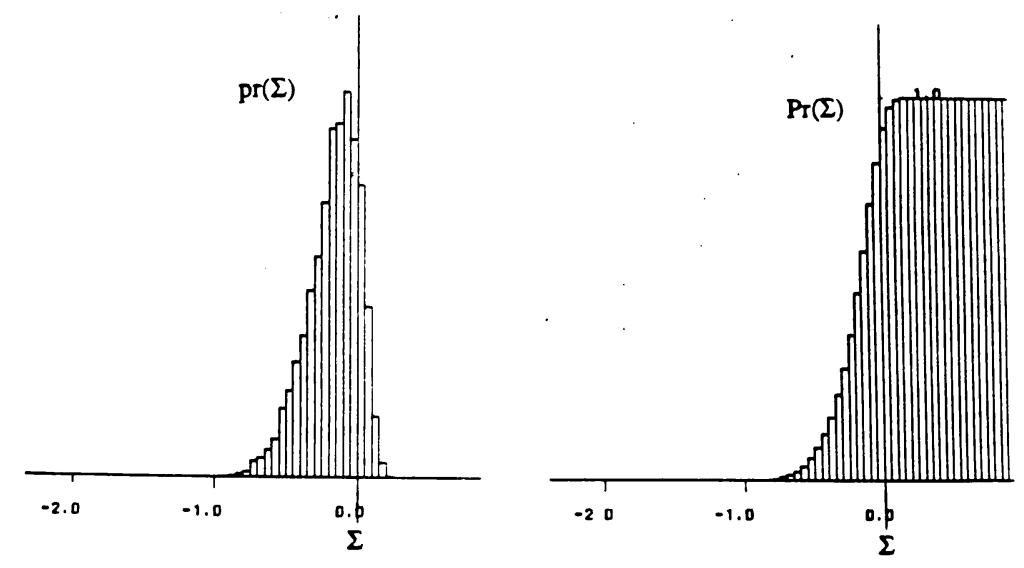

a) Case 1 .
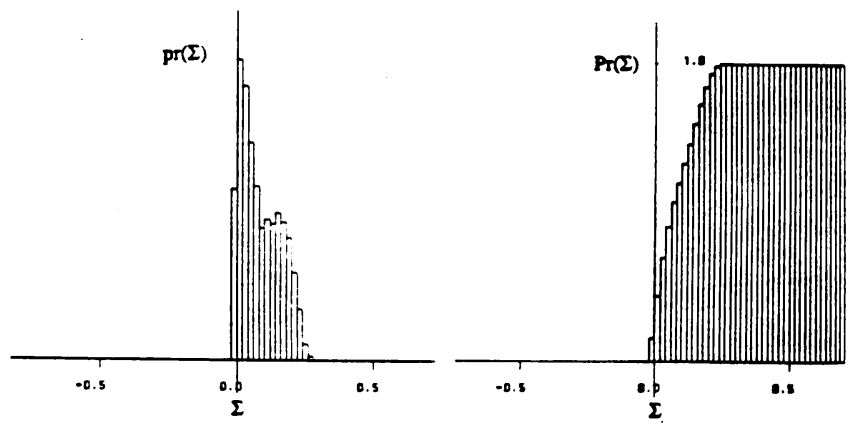

b) Case 2 .

Figure 5. Histograms and cumulative distributions for LQG counterexample, 10,000 samples, $\bar{\mu}=1, \sigma_{\mu}=0.05$. (from [28])

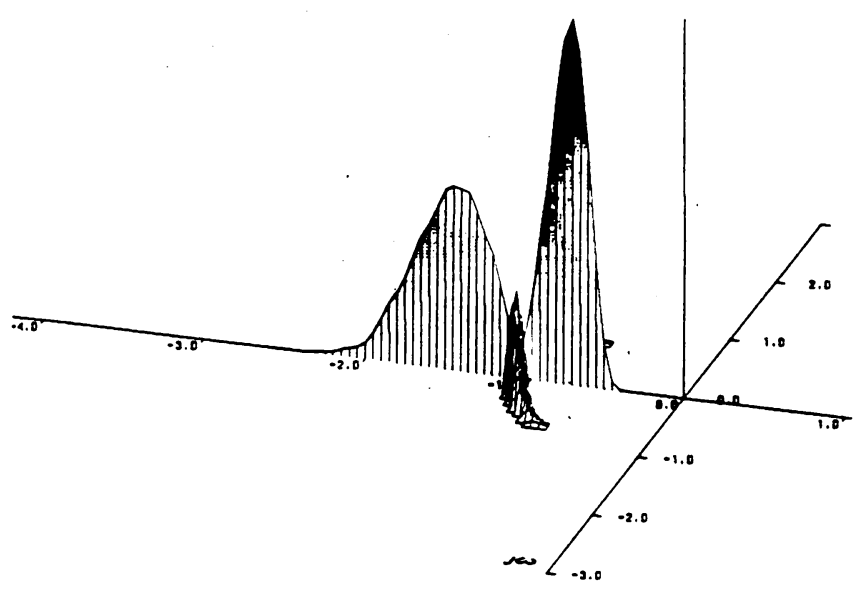

a) Case 1 LQ Regulator.

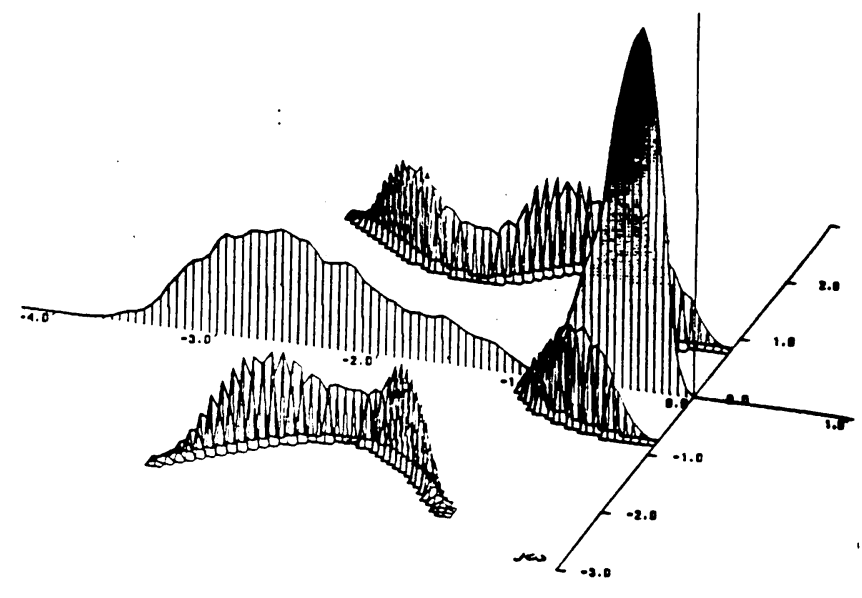

b) LQG/LTR Regulator $(v=5)$.

Figure 6. Stochastic root loci for Case $1 \mathrm{LQ}$ and LQG/LTR $(v=5)$ regulators. 
3.3 Benchmark Control Problem. Stochastic Robustness Analysis provides a "level playing field" on which to judge the robustness of controllers that have been designed by alternate methods. Ten compensators prepared for the 1990 American Control Conference Benchmark Control Problem [50] were analyzed for their probabilities of instability given three levels of parameter uncertainty [30]. The nominal plant consisted of two unit masses connected by a unit spring, with a force, $u$, on the first mass, $m_{l}$. It is regulated by feeding back the position, $x_{2}$, of the second mass, $m_{2}$, to $u$ (hence, the measurement is $z=x_{2}$ ) With unit masses and spring constant, $k$, this undamped fourth-order system has a pair of eigenvalues at the origin and another at $\pm j \sqrt{2}$. The LTI controllers were designed by a variety of methods, including $\mathrm{H}_{\infty}$ optimization, loop transfer recovery, imaginary-axis shifting, constrained optimization, structured covariance, game theory, and the internal model principle. The design solutions were single-input/single-output compensators ranging from second- to eighth-order, and while none were designed for the evaluation conducted in [30], all were intended to be robust against uncertainty in the masses and spring constant. The parameter uncertainty cases investigated in [30] were: spring constant restricted to $(0.5,2)$ [Ps1], Ps1 plus variations of the two masses in $(0.5,1.5)$ [Ps2], and Ps2 plus small variations in internal damping (always zero or positive), loop gain, and loop time delay [Ps3].

Gain margin proved to be a poor predictor of $\hat{\mathbb{P}}$ (Fig. 7) [30]. The reason derives from the observation made in the Introduction: the changing shape of the Nyquist plot is a complex function of parameter variations that is not totally captured by nominal gain (or phase) margins. This is explicitly demonstrated for three of the compensators with similar gain margins in [51]. The best and the worst of the compensators used $\mathrm{H}_{\infty}$ criteria in design, reminding us that choice of criterion alone does not assure robust design. Compensator complexity was no guarantee of low $\hat{\mathbb{P}}$ : the sixth- and eighth-order compensators had among the highest values of $\hat{\mathbb{P}}$, while a second-order design had the third lowest $\hat{\mathbb{P}}$ over the three cases. The two lowest probabilities of instability were realized with fourth-order compensators (equal to the order of the plant).

Attention has been focused on the stability robustness of continuous-time, linear, timeinvariant systems, but it is apparent that SRA principles are applicable to other types of systems using appropriate tests for stability. For discrete-time LTI systems, the stability discriminant is the unit circle about the $z$ plane origin rather than the imaginary axis in the $s$ plane. For time-varying and nonlinear systems, the Monte Carlo tests could be based on absolute stability, Lyapunov criteria (e.g., [49]), or time-domain envelopes. The last alternative forms a natural bridge to the performance robustness metrics discussed below. The 
probabilistic stability metric $\mathbb{P}$ and its confidence intervals remain the same for all of these alternatives.

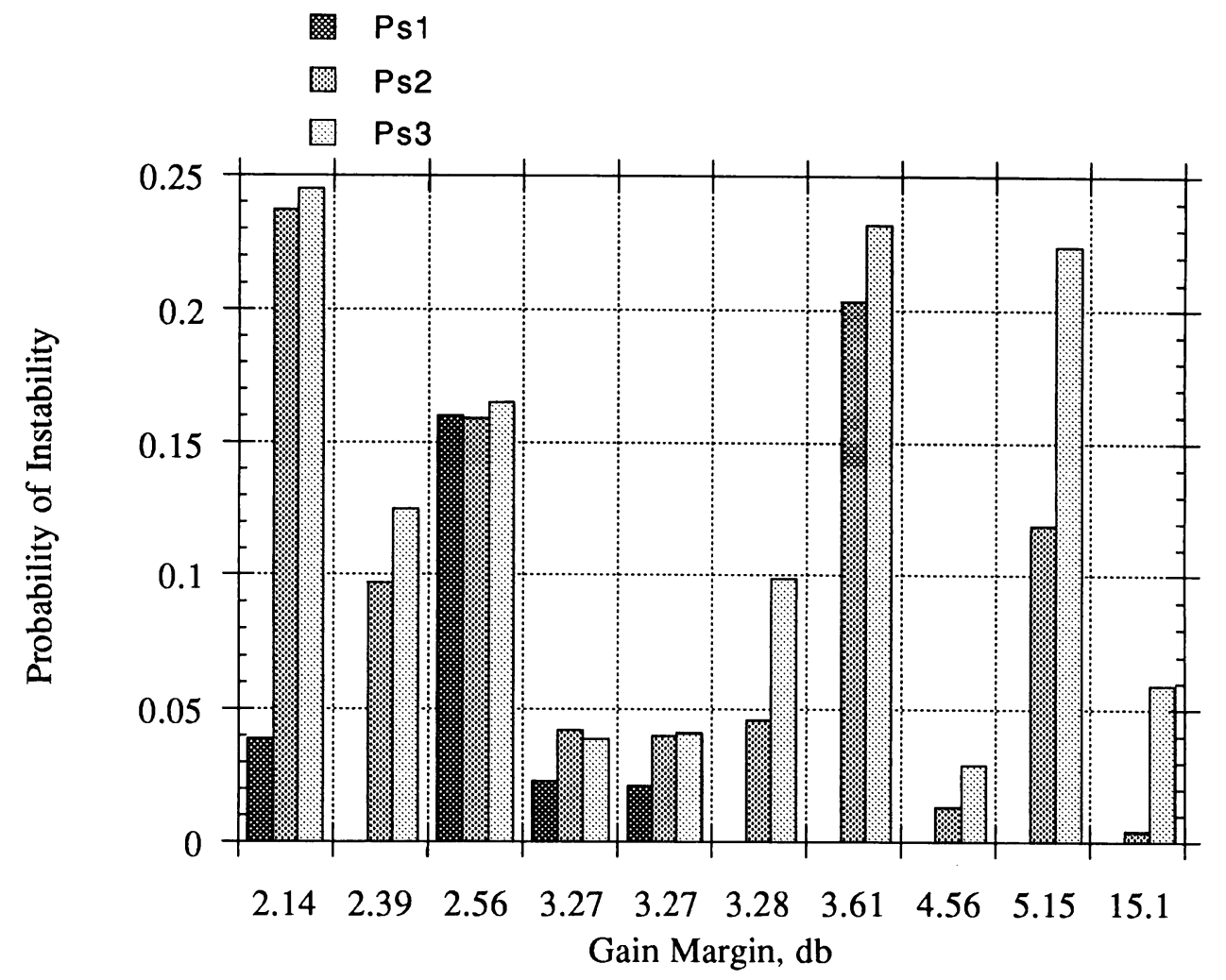

Figure 7. Probability of instability vs. gain margin for ten compensators. (from [30])

3.4 Performance Robustness. If performance metrics are expressed as envelopes of satisfactory (or acceptable) response, then the criteria take binary values, their statistics are described by binomial distributions, and the prior discussion of confidence intervals applies directly. For LTI systems, we may follow a natural progression from eigenvalue sector bounds to initial-condition transient response, step response, frequency response, and other graphical presentations, including Nyquist and Nichols charts and singular-value plots. Examples of such analyses are presented in [25, 26, 29]. Classical figures of merit can readily be introduced within the SRA framework first by identifying acceptable response envelopes, then by evaluating the probability that responses of the uncertain system will be confined to these envelopes. The Benchmark Control Problem analysis [30] included comparisons of the probabilities that settling time and control response to a unit initial disturbance would exceed acceptable values. 


\section{SYNTHESIS OF ROBUST CONTROL LAWS}

4.1 Numerical Synthesis. Design for stochastic robustness follows analysis by incorporating search. The simplest approach is to choose the best from an ensemble of controllers, without regard to the design algorithms employed for each controller. For example, given the Benchmark Control Problem, we could compare the probabilities of instability, $\mathbb{P}_{\mathrm{i}}$, excess control usage, $\mathbb{P}_{\mathrm{u}}$, and excess settling time, $\mathbb{P}_{\mathrm{Ts}}$, for the ten design solutions, selecting the one that appears most suitable. The relative importance of the three criteria must be known to make the selection, and the probability distributions of the uncertain parameters that induce them should be well motivated. Guidelines for comparing controller pairs are contained in [26].

An alternate method is to choose the parameters used in an established design procedure (e.g., LQG synthesis) so that a cost function comprised of the probabilities of unsatisfactory behavior is minimized. To test this technique, an LQG regulator was designed for the Benchmark Problem [42]. An implicit-model-following cost function produces a constantgain LQ regulator whose closed-loop dynamics are close to those of an ideal model -- a mass-spring-mass system with nominal values of $m_{1}, k$, and $m_{2}$, and with inter-mass damping added to yield an acceptable settling time $(9.6 \mathrm{sec})$. Control-rate weighting reduces peak actuator usage (adding a fifth element to the design state), and the state is estimated from the scalar output using a constant-gain Kalman filter. The settling-time limit is violated if the displacement of $m_{2}$ exceeds a \pm 0.1 -unit envelope 15 or more seconds after the disturbance. The control-usage limit is violated if control displacement exceeds one unit, and the stability requirement is violated with one or more positive closed-loop roots. Parameters are assumed to have uniform probability distributions within their ranges.

A typical design procedure has four steps:

1) Define Quadratic Cost Function of Probabilities

(4.1) $\mathrm{J}=\alpha \mathbb{P}_{\mathrm{i}}^{2}+\beta \mathbb{P}_{\mathrm{u}}^{2}+\gamma \mathbb{P}_{\mathrm{Ts}}{ }^{2}$

where $\alpha, \beta$, and $\gamma$ are scalar weights on the relative importance of instability, excess control usage, and excess settling time over the range of parameter uncertainty. $\mathbb{P}_{\mathrm{i}}, \mathbb{P}_{\mathrm{u}}$, and $\mathbb{P}_{\text {Ts }}$ are in $(0,1)$; squaring them reduces the relative significance of small values.

2) Compute Robust Linear-Ouadratic Regulator

$$
v=-\mathbf{C} \chi=-\mathbf{C}[\hat{\mathbf{x}} u]
$$

$$
u=\int v d t
$$


where $v$ is the scalar control rate, $\mathbf{C}$ is the $(1 \times 5)$ LQ gain matrix for implicit model following with control-rate weighting, and $\chi$ contains the estimate of the augmented state, $\hat{\mathbf{x}}$, and the control displacement, $u$. The control rate $v$ must be integrated to yield the original control variable $u$; hence, (4.2) and (4.3) have a low-pass filtering effect on the control. A reduced-sample Monte Carlo evaluation is made at each search point. $\mathrm{J}$ is then minimized with respect to the LQ design parameters and the ideal model parameters.

The cost function forms a hypersurface adjoined to the design parameter space. The surface is non-smooth because there is uncertainty in the results of Monte Carlo evaluation. Furthermore, the surface has plateaus where probabilities are one or zero. For a fixed number of evaluations, there is a tradeoff between the number of design points and the quality of the cost estimate at each point. Parameters are adjusted in a series of line searches. These line searches are probabilistic, as only a few evaluations (125) are made at each design point; hence, the function estimate is "noisy."

The Kolmogorov-Smirnov 2-sample test identifies statistically significant trends [57]. If no trend is found, the search moves to the next parameter. Otherwise the cost function is smoothed, and the minimum-cost point defines the new nominal value for that parameter. Having searched all parameters, the search ranges are halved, and the cycle is repeated. The search continues until there is no further improvement; typically this takes two or three cycles.

\section{3) Define Robust Kalman Filter}

$$
\dot{\hat{\mathbf{x}}}=\mathbf{F} \hat{\mathbf{X}}+\mathbf{G} u+\mathbf{K}(z-\mathbf{H} \hat{\mathbf{x}})
$$

where $\hat{\mathbf{x}}$ is the estimated state, $u$ is the control, $z$ is the scalar output, $\mathbf{F}, \mathbf{G}, \mathbf{K}$, and $\mathbf{H}$ are system, control, estimator gain, and output matrices. The disturbance-effect matrix used in design accounts for the "process noise" arising from parameter variations in the closed-loop system. The initial Kalman filter is designed with assumed models of the disturbance and noise. Process noise due to plant variations is estimated by propagating an equivalent discrete-time model of the closed-loop system. The estimation is based on the residual,

$$
\begin{aligned}
\mathbf{q}_{\mathbf{k}} & =\hat{\mathbf{x}}_{\mathrm{k}+1}-\Phi_{\mathrm{f}} \hat{\mathbf{x}}_{\mathbf{k}}-\Gamma_{\mathrm{f}} u_{k} \\
& =\Lambda w_{k}+\left(\Phi_{\mathrm{a}}-\Phi_{\mathrm{f}}\right) \hat{\mathbf{x}}_{\mathbf{k}}+\left(\Gamma_{\mathrm{a}}-\Gamma_{\mathrm{f}}\right) u_{k}
\end{aligned}
$$

where $w_{k}$ is the disturbance. $\Phi_{\mathrm{a}}$ and $\Phi_{\mathrm{f}}$ are the plant and filter models of the state transition matrix, $\Gamma_{\mathrm{a}}$ and $\Gamma_{\mathrm{f}}$ are the control effect matrices, and $\Lambda$ is the plant disturbance effect 
matrix. With parameter variation, the residual includes fictitious disturbances due to the difference between effects propagated by the plant and the filter. The residuals are found by Monte Carlo evaluation of responses to disturbance and control. The plant model is changed by random-number generators during the Monte Carlo evaluation, and the process-noise covariance is estimated by batch-processing the residuals according to [58]:

$$
\mathrm{Q}=\frac{1}{\mathrm{~N}-1} \sum_{\mathrm{k}=1}^{\mathrm{N}} \mathbf{q}_{\mathrm{k}} \mathbf{q}_{\mathrm{k}}{ }^{\mathrm{T}}
$$

The measurement-noise matrix is calculated similarly. The filter designed using this disturbance matrix rejects the effects caused by differences between the plant and the filter model; hence, it is more robust than the initial filter.

\section{4) Perform Stochastic Robustness Analysis of the Closed-Loop System}

Stochastic robustness of the LQG-controlled plant is evaluated, and the above steps are repeated if necessary. The final design is produced by a search that minimizes the cost function with respect to all control and estimator design parameters.

4.2 Results of Design. As a trial, four compensators were formulated with a search procedure that used about 80,000 trials for each design. The first three emphasized $\mathbb{P}_{\mathrm{i}}, \mathbb{P}_{\mathrm{u}}$, and $\mathbb{P}_{\mathrm{Ts}}$, respectively; the fourth emphasized $\mathbb{P}_{\mathrm{i}}$ but placed greater weight on $\mathbb{P}_{\mathrm{u}}$ and $\mathbb{P}_{\text {Ts. }}$ The compensator transfer functions, $H_{z u}$, are as follows:

$$
\begin{aligned}
& H_{z u}=\frac{79.3(\mathrm{~s}-0.8)(\mathrm{s}+0.57)(\mathrm{s}+0.11)}{\left[\mathrm{s}^{2}+2(0.60)(3.2)+(3.2)^{2}\right]\left[\mathrm{s}^{2}+2(0.93)(3.7)+(3.7)^{2}\right][\mathrm{s}+0.46]} \\
& H_{z u}=\frac{3.16(\mathrm{~s}-3.5)(\mathrm{s}+5.6)(\mathrm{s}+0.14)}{\left[\mathrm{s}^{2}+2(0.58)(3.4)+(3.4)^{2}\right]\left[\mathrm{s}^{2}+2(0.65)(2.6)+(2.6)^{2}\right][\mathrm{s}+1.18]} \\
& H_{z u}=\frac{8.2(\mathrm{~s}-4.7)(\mathrm{s}+3.9)(\mathrm{s}+0.24)}{\left[\mathrm{s}^{2}+2(0.63)(3.7)+(3.7)^{2}\right]\left[\mathrm{s}^{2}+2(0.58)(2.7)+(2.7)^{2}\right][\mathrm{s}+1.6]} \\
& H_{z u}=\frac{5.42(\mathrm{~s}-2.9)(\mathrm{s}+4.0)(\mathrm{s}+0.15)}{\left[\mathrm{s}^{2}+2(0.67)(4.0)+(4.0)^{2}\right]\left[\mathrm{s}^{2}+2(0.51)(2.0)+(2.0)^{2}\right][\mathrm{s}+1.36]}
\end{aligned}
$$

Each of the compensators is strictly proper and contains a non-minimum-phase zero. Robustness costs for both the robust LQ and LQG regulators, evaluated with 20,000 trials, are shown in Table 1, as are the associated probabilities. Adding an estimator to the LQ design to form an LQG regulator increases the robustness cost in every case. 
TABLE 1

Robustness Costs and Probabilities of Constraint Violation for

Four Robust LQG Controllers

Case 1

$\alpha, \beta, \gamma$
LQ Cost
LQG Cost
$\hat{\mathbb{P}}_{\mathrm{i}}$
$\hat{\mathbb{P}}_{\mathrm{u}}$
$\hat{\mathbb{P}}_{\mathrm{Ts}}$

$1,0.01,0.01$

0.0002

0.0059

0.0034

0.1077

0.7588
Case 2

$$
0.01,1,0.01
$$

0.0004

0.0045

0.0173

0.0173

0.6504
Case 3

$$
0.01,0.01,1
$$

0.0081

0.2249

0.2953

0.3591

0.4719
Case 4

$1,0.02,0.06$

0.0014

0.0157

0.0671

0.0671

0.4305

Not surprisingly, the ten compensators evaluated in [30], which were not designed to these criteria, have larger robustness costs than the robust LQG designs in all but a single case. One of the earlier designs had lower cost for Case 3, achieving low settling-time violation by always violating our imposed control limit by about two orders of magnitude. (Large exceedances were not penalized more than small exceedances; hence $\mathbb{P}_{\mathrm{T}}$ was one, but it was weighted by a small value.)

Nominal disturbance responses of the four newly designed compensators are shown in Fig. 8. Each of these compensators can be claimed to be "robust" according to different stability and performance criteria. In all cases, $x_{2}$ satisfies the settling-time requirements, though the response shapes are quite different. The maximum control usage is 0.82 units for Case 3.

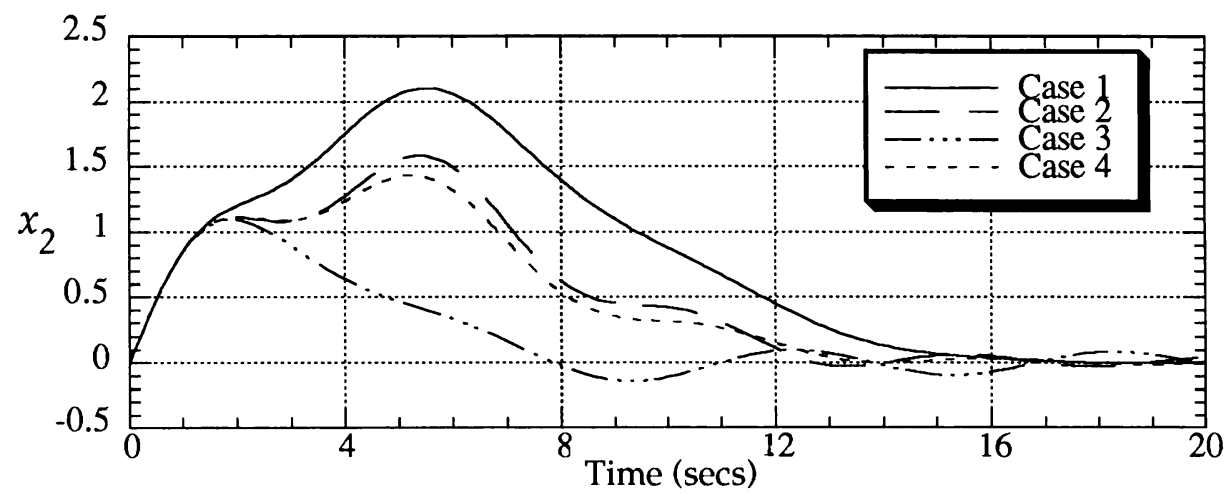

Figure 8. Output Responses to Unit Disturbance Inputs for Linear-Quadratic-Gaussian Regulators Designed to Minimize Four Stochastic Robustness Costs. 
Three additional compensators were designed with the Case 4 cost function, two to assess possibilities for reducing compensator complexity and one to evaluate effects of loop transfer recovery. Case 5 eliminated control-rate weighting and the associated low-pass filter, producing a fourth-order compensator with $\left(\hat{\mathbb{P}}_{\mathrm{i}}, \hat{\mathbb{P}}_{\mathrm{u}}, \hat{\mathbb{P}}_{\mathrm{Ts}}\right)=(0.1108,0.115,0.6675)$. All probabilities are higher than those of Case 4. Case 6 further reduced the order to three by residualizing the filter's dynamic model, yielding $\left(\hat{\mathbb{P}}_{\mathrm{i}}, \hat{\mathbb{P}}_{\mathrm{u}}, \hat{\mathbb{P}}_{\mathrm{Ts}}\right)=(0,0,1)$. The minimization identified an excellent design for stability and control usage, but the settling time specification was never met. Because the search is made over a probabilistic surface, it is possible that this result was produced by a local minimum. Case 7 was a fifth-order compensator in which loop transfer recovery was applied with $v=10^{12}$, giving $\left(\hat{\mathbb{P}}_{\mathrm{i}}, \hat{\mathbb{P}}_{\mathrm{u}}, \hat{\mathbb{P}}_{\mathrm{Ts}}\right)=$ $(0.078,1,0.166)$. Excellent stability and settling-time properties were achieved through excessive control usage.

Nominal characteristics of the seven LQG compensators are summarized in Table 2. There is a broad range of stability margins and responses, yet each design can claim to be robust by some stochastic measure. As in [30], there is little direct correlation of gain margins (GM) and phase margins (PM) with the probability of instability; both low and high values of the stability margins can be associated with significant $\hat{\mathbb{P}}_{\mathrm{i}}$. Nominal settling

time and $\hat{\mathbb{P}}_{\text {Ts }}$ are loosely correlated, as are nominal control usage and $\hat{\mathbb{P}}_{\mathrm{u}}$, though there is little correlation in nominal control and state peak responses. The LQG/LTR regulator (Case 7) is seen to possess the highest gain and phase margins, but its probabilities of unsatisfactory behavior are high and its control usage is excessive.

TABLE 2

Nominal Characteristics of Seven Robust LQG Compensators

Nominal Stability Margins

\begin{tabular}{|c|c|c|c|c|c|c|c|}
\hline$\underline{\text { Case }}$ & $\underline{1}$ & $\underline{2}$ & $\underline{3}$ & $\underline{4}$ & $\underline{5}$ & $\underline{6}$ & $\underline{7}$ \\
\hline $\mathrm{GM}(\mathrm{dB})$ & 4.45 & 5.1 & 0.59 & 3.97 & 3.28 & 2.48 & 11.5 \\
\hline PM (deg) & 28.7 & 31.2 & 21.5 & 32 & 23.6 & 26.1 & 39 \\
\hline \multicolumn{8}{|c|}{ Nominal Unit Disturbance Response } \\
\hline $\mathrm{T}_{\mathrm{S}}(\mathrm{sec})$ & 14.4 & 12.1 & 10.1 & 12.5 & 14.2 & 30.5 & 8.5 \\
\hline$u_{\max }$ & 0.59 & 0.46 & 0.82 & 0.54 & 0.54 & 0.53 & 57.55 \\
\hline$x_{2 \max }$ & 2.12 & 1.59 & 1.09 & 1.43 & 1.07 & 1.07 & 0.57 \\
\hline \multicolumn{8}{|c|}{ Nominal Unit Measurement-Error Control Response } \\
\hline$u_{\max }$ & 4.25 & 0.86 & 1.75 & 0.99 & 0.57 & 0.63 & 37170 \\
\hline
\end{tabular}




\section{CONCLUSION}

Stochastic robustness provides a sound basis for analyzing and designing robust control systems. Its criteria are direct extensions of classical design criteria that are closely related to design goals and to practical characterization of parametric uncertainty. Consequently, it is possible to merge the probabilistic point of view with a variety of preexisting analysis and design techniques. The current analysis reveals new insights about the robustness of linear-quadratic-Gaussian regulators, the significance of gain and phase margins as predictors of robustness, and the relationships between nominal system characteristics and the likelihood of satisfactory system behavior. In any given problem, there is not a single robust solution but a family of solutions that depend on both subjective and objective assessment. The synthesis approach presented here recognizes that different criteria (e.g., probabilities of excessive settling time, control usage, and instability) may have greater relative importance in different settings, allowing tradeoffs to be made among competing design requirements.

\section{REFERENCES}

[1] F. KOZIN, A Survey of Stability of Stochastic Systems, Automatica, 5, 1969, pp. 95-112.

[2] H. J. KUSHNER, Stochastic Stability and Control, Academic Press, New York, 1967.

[3] E. YAZ, Deterministic and Stochastic Robustness Measures for Discrete Systems, IEEE Trans. Auto. Cont., 33 (10), 1988, pp. 952-955.

[4] K. ITO, On Stochastic Differential Equations, Mem. Am. Math. Soc., 4, 1951.

[5] P. I. KUZNETSOV, R. L. STRATONOVICH, AND V. I. TIKHONOV, Non-Linear Transformations of Stochastic Processes, Pergamon Press, Oxford, 1965.

[6] M. COTTRELL, J.-C. FORT, AND G. MALGOUYRES, Large Deviations and Rare Events in the Study of Stochastic Algorithms, IEEE Trans. Auto. Cont., AC-28 (9), 1983, pp. 907-920.

[7] K. B. LIM AND J. L. JUNKINS, Probability of Stability: New Measures of Stability Robustness for Linear Dynamical Systems, J. Astro. Sci., 35 (4), 1987, pp. 383-397.

[8] C. PIERRE, Root Sensitivity to Parameter Uncertainties: a Statistical Approach, Int. J. Control, 49 (2), 1989, pp. 521-532.

[9] B. S. MORGAN, JR., Sensitivity Analysis and Synthesis of Multivariable Systems, IEEE Trans. Auto. Cont., AC-11 (4), 1966, pp. 506-512.

[10] E. J. DAVISON AND A. GOLDENBERG, Robust Control of a General Servomechanism, Automatica, 11 (5), 1975, pp. 461-471.

[11] B. C. MOORE, On the Flexibility Offered By State Feedback in Multivariable Systems Beyond Closed Loop Eigenvalue Assignment, IEEE Trans. Auto. Cont., AC-21 (5), 1976, pp. 689-692.

[12] J. W. HOWZE AND R. K. CAVIN, III, Regulator Design with Modal Insensitivity, IEEE Trans. Auto. Cont., AC-24 (3), 1979, pp. 466-469.

[13] R. F. STENGEL, Some Effects of Parameter Variations on the Lateral-Directional Stability of Aircraft, J. Guid. Cont., 3 (2), 1980, pp. 124-131.

[14] E. G. GILBERT, Conditions for Minimizing the Norm Sensitivity of Characteristic Roots, IEEE Trans. Auto. Cont., AC-29 (7), 1984, pp. 658-661.

[15] R. F. STENGEL, Stochastic Optimal Control: Theory and Application, J. Wiley \& Sons, New York, 1986.

[16] A. PAPOULIS, Probability \& Statistics, Prentice-Hall, Englewood Cliffs, 1990.

[17] I. MILLER AND J. E. FREUND, Probability and Statistics for Engineers, Prentice-Hall, Englewood Cliffs, 1977. 
[18] G. W. BROWN, Monte Carlo Methods, in Modern Mathematics for the Engineer, E. F. Beckenbach, ed., McGraw-Hill, New York, 1956.

[19] E. J. HENLEY AND H. KUMAMOTO, Probabilistic Risk Assessment: Reliability Engineering, Design, and Analysis, IEEE Press, New York, 1992.

[20] G. APOSTOLAKIS, The Concept of Probability in Safety Assessments of Technological Systems, Science, 250, 1990, pp. 1359-1364.

[21] F. C. SCHWEPPE, Uncertain Dynamic Systems, Prentice-Hall, Englewood Cliffs, 1973.

[22] P. S. MAYBECK, Stochastic Models, Estimation, and Control, 1, Academic Press, Englewood Cliffs, 1979.

[23] R. F. STENGEL AND L. R. RAY, Stochastic Robustness of Linear-Time-Invariant Control Systems, IEEE Trans. Auto. Cont., AC-36 (1), 1991, pp. 82-87.

[24] L. R. RAY AND R. F. STENGEL, Application of Stochastic Robustness to Aircraft Control Systems, J. Guid. Cont. Dyn., 14 (6), 1991, pp. 1251-1259.

[25] L. R. RAY AND R. F. STENGEL, Stochastic Stability and Performance Robustness of Linear Multivariable Systems, Proc. Am. Cont. Conf., 1990, pp. 462-467.

[26] L. R. RAY AND R. F. STENGEL, Stochastic Performance Robustness of Aircraft Control Systems, Proc. AIAA Guid. Nav. Cont. Conf., 1990, pp. 863-873 (to appear).

[27] L. R. RAY AND R. F. STENGEL, Computer-Aided Analysis of Linear Control System Robustness, Proc. 29th IEEE Conf. Dec. Cont., 1990, pp. 3468-3469 (to appear).

[28] L. R. RAY, Stochastic Robustness of Linear Multivariable Control Systems: Towards Comprehensive Robustness Analysis, Princeton University, Ph. D. Thesis MAE-1902T, 1991.

[29] L. R. RAY AND R. F. STENGEL, A Monte Carlo Approach to the Analysis of Control System Robustness (to appear).

[30] R. F. STENGEL AND C. I. MARRISON, Robustness of Solutions to a Benchmark Control Problem, J. Guid. Cont. Dyn., 15 (5), 1992, pp. 1060-1067.

[31] L. EL GHAOUI AND A. E. BRYSON, JR., Worst Case Parameter Changes for Stabilized Conservative SISO Systems, AIAA-91-2767-CP, 1991.

[32] K. A. WISE, Comparison of Six Robustness Tests Evaluating Missile Autopilot Robustness to Uncertain Aerodynamics, J. Guid, Cont. Dyn., 15 (4), 1992, pp. 861-870.

[33] R. C. SPEAR, The Application of Kolmogorov-Rényi Statistics to Problems of Parameter Uncertainty in Systems Design, Int. J. Cont., 11 (5), 1970, pp. 771-778.

[34] D. M. AUSLANDER, R. C. SPEAR, AND G. E. YOUNG, A Simulation-Based Approach to the Design of Control Systems With Uncertain Parameters, J. Dyn. Sys. Meas. Cont., 104 (1), 1982, pp. 20-26.

[35] G. E. YOUNG AND D. M. AUSLANDER, A Design Methodology for Nonlinear Systems Containing Parameter Uncertainty, J. Dyn. Sys. Meas. Cont, 106 (3), 1984, pp. 15-20.

[36] K. C. Q. TSAI AND D. M. AUSLANDER, A Statistical Methodology of Designing Controllers for Minimum Sensitivity of Parameter Variations, J. Dyn. Sys. Meas. Cont., 110 (6),1984, pp. 126133.

[37] A. A. SCHY AND D. P. GEISY, Multiobjective Insensitive Design of Airplane Control Systems with Uncertain Parameters, Proc. AIAA Guid. Nav. Cont. Conf., 1981.

[38] D. D. SILJAK, Parameter Space Methods for Robust Control Design: A Guided Tour, Univ. of Cal., Santa Clara, EECS-031588, 1988.

[39] J. ACKermanN, D. KAESbaUer, AND W. SIENEl, Design by Search, $1^{\text {st }}$ IFAC Symp. Des. Meth. Cont. Sys., 1991.

[40] L. R. ANDERSON, Fine Tuning of Aircraft Control Laws using Pro-Matlab Software, AIAA-912600-CP, 1991.

[41] K. KRISHNAKUMAR, AND D. E. GOLDBERG, Control System Optimization Using Genetic Algorithms, J. Guid. Cont. Dyn., 15 (3), 1992, pp.735-740.

[42] R. F. STENGEL AND C. I. MARRISON, Stochastic Robustness Synthesis for a Benchmark Problem, Proc. Amer. Cont. Conf., Chicago, 1992, pp. 2421-2422.

[43] W. J. CONOVER, Practical Non-Parametric Statistics, J. Wiley \& Sons, New York, 1980.

[44] T. W. ANDERSON AND H. BURNSTEIN, Approximating the Upper Binomial Confidence Limit, J. Amer. Stat. Assoc., 62, 1967, pp. 857-861.

[45] T. W. ANDERSON AND H. BURNSTEIN, Approximating the Lower Binomial Confidence Limit, J. Amer. Stat. Assoc., 63, 1968, pp. 1413-1415.

[46] J. C. DOYLE, Guaranteed Margins for LQG Regulators, IEEE Trans. Auto. Cont., AC-23 (4), 1978, pp. 756-757. 
[47] J. C. DOYLE AND G. STEIN, Robustness with Observers, IEEE Trans. Auto. Cont, AC-24 (4), 1979, pp. 6-7-611.

[48] N. A. LEHTOMAKI, N. R. SANDELL, JR., AND M. ATHANS, Robustness Results in LinearQuadratic Gaussian Based Multivariable Control Designs, IEEE Trans. Auto. Cont., AC-26 (1), 1981, pp. 75-92.

[49] K. S. NARENDRA AND J. H. TAYLOR, Frequency Domain Criteria for Absolute Stability, Academic Press, New York, 1973.

[50] B. WIE AND D. S. BERNSTEIN, A Benchmark Problem for Robust Control Design, Proc. Am. Cont. Conf., 1990, pp. 961-962.

[51] C. I. MARRISON AND R. F. STENGEL, Gain and Phase Margins as Indicators of Robustness, Proc. IEEE Reg. Cont. Conf., New York, 1992, pp. 5-8.

[52] K. OGATA, Modern Control Engineering, Prentice-Hall, Englewood Cliffs, 1990.

[53] Y. TAKAHASHI, M. J. RABINS, AND D. M. AUSLANDER, Control and Dynamic Systems, AddisonWesley, Reading, 1972.

[54] H. W. BODE, Network Analysis and Feedback Amplifier Design, Van Nostrand, Princeton, 1945.

[55] R. OLDENBURGER, ed., Frequency Response, Macmillan, New York, 1936.

[56] R. K. YEDAVALLI, S. S. BANDA, AND D. B. RIDGELY, Time-Domain Stability Robustness Measures for Linear Regulators, J. Guid. Cont. Dyn., 8 (4), 1985, pp. 520-524.

[57] L. SACHS, Applied Statistics: A Handbook of Techniques, Springer-Verlag, New York, 1984.

[58] K. A. MYERS AND B. D. TAPLEY, Adaptive Sequential Estimation with Unknown Noise Statistics, IEEE Trans. Auto. Cont., AC-21(4), 1976, pp. 520-523. 


\section{Recent IMA Preprints}

IMA Summer Program for Graduate Students, Mathematical Modeling

Wayne Barrett, Charles R. Johnson, and Pablo Tarazaga, The real positive definite completion problem for a simple cycle

Charles A. McCarthy, Fourth order accuracy for a cubic spline collocation method

Martin Hanke, James Nagy, and Robert Plemmons, Preconditioned iterative regularization for I11-posed problems

John R. Gilbert, Esmond G. Ng, and Barry W. Peyton, An efficient algorithm to compute row and column counts for sparse Cholesky factorization

Xinfu Chen, Existence and regularity of solutions of a nonlinear nonuniformly elliptic system arising from a thermistor problem

Xinfu Chen and Weiqing Xie, Discontinuous solutions of steady state, viscous compressible Navier-Stokes equations

E.G. Kalnins, Willard Miller, Jr., and Sanchita Mukherjee, Models of $q$-algebra representations: Matrix elements of the $q$-oscillator algebra

W. Miller, Jr. and Lee A. Rubel, Functional separation of variables for Laplace equations in two dimensions

I. Gohberg and I. Koltracht, Structured condition numbers for linear matrix structures

Xinfu Chen, Hele-Shaw problem and area preserved curve shortening motion

Zhangxin Chen and Jim Douglas, Jr. Modelling of compositional flow in naturally fractured reservoirs

Harald K. Wimmer, On the existence of a least and negative-semidefinite solution of the discrete-time algebraic Riccati equation

Harald K. Wimmer, Monotonicity and parametrization results for continuous-time algebraic Riccati equations and Riccati inequalities

Bart De Moor, Peter Van Overschee, and Geert Schelfhout, $H_{2}$ model reduction for SISO systems

Bart De Moor, Structured total least squares and $L_{2}$ approximation problems

Chjan Lim, Nonexistence of Lyapunov functions and the instability of the Von Karman vortex streets

David C. Dobson and Fadil Santosa, Resolution and stability analysis of an inverse problem in electrical impedance tomography - dependence on the input current patterns

C.N. Dawson, C.J. van Duijn, and M.F. Wheeler, Characteristic-Galerkin methods for contaminant transport with non-equilibrium adsorption kinetics

Bing-Yu Zhang, Analyticity of solutions of the generalized Korteweg-de Vries equation with respect to their initial values

Neerchal K. Nagaraj and Wayne A. Fuller, Least squares estimation of the linear model with autoregressive errors

H.J. Sussmann \& W. Liu, A characterization of continuous dependence of trajectories with respect to the input for control-affine systems

Karen Rudie \& W. Murray Wonham, Protocol verification using discrete-event systems

Rohan Abeyaratne \& James K. Knowles, Nucleation, kinetics and admissibility criteria for propagating phase boundaries

Gang Bao \& William W. Symes, Computation of pseudo-differential operators

Srdjan Stojanovic, Nonsmooth analysis and shape optimization in flow problem

Miroslav Tuma, Row ordering in sparse $Q R$ decomposition

Onur Toker \& Hitay Özbay, On the computation of suboptimal $H^{\infty}$ controllers for unstable infinite dimensional systems

Hitay Özbay, $H^{\infty}$ optimal controller design for a class of distributed parameter systems

J.E. Dunn \& Roger Fosdick, The Weierstrass condition for a special class of elastic materials

Bei Hu \& Jianhua Zhang, A free boundary problem arising in the modeling of internal oxidation of binary alloys

Eduard Feireisl \& Enrique Zuazua, Global attractors for semilinear wave equations with locally distributed nonlinear damping and critical exponent

I-Heng McComb \& Chjan C. Lim, Stability of equilibria for a class of time-reversible, $D_{n} x O(2)$-symmetric homogeneous vector fields

Ruben D. Spies, A state-space approach to a one-dimensional mathematical model for the dynamics of phase transitions in pseudoelastic materials

H.S. Dumas, F. Golse, and P. Lochak, Multiphase averaging for generalized flows on manifolds

Bei Hu \& Hong-Ming Yin, Global solutions and quenching to a class of quasilinear parabolic equations

Zhangxin Chen, Projection finite element methods for semiconductor device equations

Peter Guttorp, Statistical analysis of biological monitoring data

Wensheng Liu \& Héctor J. Sussmann, Abnormal sub-Riemannian minimizers 
Chjan C. Lim, A combinatorial perturbation method and Arnold's whiskered Tori in vortex dynamics

Yong Liu, Axially symmetric jet flows arising from high speed fiber coating

Li Qiu \& Tongwen Chen, $\mathcal{H}_{2}$ and $\mathcal{H}_{\infty}$ designs of multirate sampled-data systems

Eduardo Casas \& Jiongmin Yong, Maximum principle for state-constrained optimal control problems covered by quasilinear elliptic equations

Suzanne M. Lenhart \& Jiongmin Yong, Optimal control for degenerate parabolic equations with logistic growth

1065

1066

1067

1068

1069

1070

1071

1072

1073

1074

1075

1076

1077

1078

1079

1080

1081

1082

1083

1084

1085

1086

1087

1088

1089

1090

1091

1092

1093

1094

1095

1096

1097

1098

1099

1100

1101

1102

1103

1104

Suzanne Lenhart, Optimal control of a convective-diffusive fluid problem

Enrique Zuazua, Weakly nonlinear large time behavior in scalar convection-diffusion equations

Caroline Fabre, Jean-Pierre Puel \& Enrike Zuazua, Approximate controllability of the semilinear heat equation

M. Escobedo, J.L. Vazquez \& Enrike Zuazua, Entropy solutions for diffusion-convection equations with partial diffusivity

M. Escobedo, J.L. Vazquez \& Enrike Zuazua, A diffusion-convection equation in several space dimensions

F. Fagnani \& J.C. Willems, Symmetries of differential systems

Zhangxin Chen, Bernardo Cockburn, Joseph W. Jerome \& Chi-Wang Shu, Mixed-RKDG finite element methods for the 2-D hydrodynamic model for semiconductor device simulation

M.E. Bradley \& Suzanne Lenhart, Bilinear optimal control of a Kirchhoff plate

Héctor J. Sussmann, A cornucopia of abnormal subriemannian minimizers. Part I: The four-dimensional case

Marek Rakowski, Transfer function approach to disturbance decoupling problem

Yuncheng You, Optimal control of Ginzburg-Landau equation for superconductivity

Yuncheng You, Global dynamics of dissipative modified Korteweg-de Vries equations

Mario Taboada \& Yuncheng You, Nonuniformly attracting inertial manifolds and stabilization of beam equations with structural and Balakrishnan-Taylor damping

Michael Böhm \& Mario Taboada, Global existence and regularity of solutions of the nonlinear string equation

Zhangxin Chen, BDM mixed methods for a nonlinear elliptic problem

J.J.L. Velázquez, On the dynamics of a closed thermosyphon

Frédéric Bonnans \& Eduardo Casas, Some stability concepts and their applications in optimal control problems

Hong-Ming Yin, $\mathcal{L}^{2, \mu}(Q)$-estimates for parabolic equations and applications

David L. Russell \& Bing-Yu Zhang, Smoothing and decay properties of solutions of the Korteweg-de Vries equation on a periodic domain with point dissipation

J.E. Dunn \& K.R. Rajagopal, Fluids of differential type: Critical review and thermodynamic analysis

Mary Elizabeth Bradley \& Mary Ann Horn, Global stabilization of the von Kármán plate with boundary feedback acting via bending moments only

Mary Ann Horn \& Irena Lasiecka, Global stabilization of a dynamic von Kármán plate with nonlinear boundary feedback

Vilmos Komornik, Decay estimates for a petrovski system with a nonlinear distributed feedback

Jesse L. Barlow, Perturbation results for nearly uncoupled Markov chains with applications to iterative methods

Jong-Shenq Guo, Large time behavior of solutions of a fast diffusion equation with source

Tongwen Chen \& $\mathbf{L i}$ Qiu, $\mathcal{H}_{\infty}$ design of general multirate sampled-data control systems

Satyanad Kichenassamy \& Walter Littman, Blow-up surfaces for nonlinear wave equations, I

Nahum Shimkin, Asymptotically efficient adaptive strategies in repeated games, Part I: certainty equivalence strategies

Caroline Fabre, Jean-Pierre Puel \& Enrique Zuazua, On the density of the range of the semigroup for semilinear heat equations

Robert F. Stengel, Laura R. Ray \& Christopher I. Marrison, Probabilistic evaluation of control system robustness

H.O. Fattorini \& S.S. Sritharan, Optimal chattering controls for viscous flow

Kathryn E. Lenz, Properties of certain optimal weighted sensitivity and weighted mixed sensitivity designs

Gang Bao \& David C. Dobson, Second harmonic generation in nonlinear optical films

Avner Friedman \& Chaocheng Huang, Diffusion in network

Xinfu Chen, Avner Friedman \& Tsuyoshi Kimura, Nonstationary filtration in partially saturated porous media

Walter Littman \& Baisheng Yan, Rellich type decay theorems for equations $P(D) u=f$ with $f$ having support in a cylinder

Satyanad Kichenassamy \& Walter Littman, Blow-up surfaces for nonlinear wave equations, II

Nahum Shimkin, Extremal large deviations in controlled I.I.D. processes with applications to hypothesis testing

A. Narain, Interfacial shear modeling and flow predictions for internal flows of pure vapor experiencing film condensation

Andrew Teal \& Laurent Praly, Global stabilizability and observability imply semi-global stabilizability by output feedback 